\title{
Isolation and Mapping of Mu nu Mutants Which Grow in him Mutants of E. coli
}

\author{
Roberta K. Yoshida, ${ }^{*}$ Janet L. Miller, ${ }^{*, 1}$ Harvey I. Miller, ${ }^{+2}$ \\ David I. Friedman, $\dagger$ and Martha M. Howe ${ }^{+3}$
}

\begin{abstract}
*Department of Bacteriology, University of Wisconsin, Madison, Wisconsin 53706, and $†$ Department of Microbiology and Immunology, University of Michigan, Ann Arbor, Michigan 48109
\end{abstract}

Received March 1, 1982; accepted March 22, 1982

\begin{abstract}
Mu $n u A$ and $\mathrm{Mu} n u B$ mutants were selected by their ability to form plaques on lawns of Escherichia coli himA and himB mutants, respectively. Deletion mapping of the $n u A$ and $n u B$ mutations by marker rescue from $\lambda \mathrm{pMu}$ transducing phages or from hosts containing deleted $\mathrm{Mu}$ prophages indicated that $n u A$ mutations are located within or to the left of gene $A$ and that $n u B$ mutations are located within or between $\mathrm{Mu}$ genes $G$ and $I$.
\end{abstract}

One approach to defining the interactions between a phage and its bacterial host is to isolate and characterize host mutants that inhibit some aspect of phage development and then phage mutants which overcome that inhibition. For temperate phage $\mathrm{Mu}$, Escherichia coli K12 $d n a B, d n a C$, and possibly $d n a E$ functions are needed for phage replication (1), and host RNA polymerase is required for phage transcription (2). Mu also requires host functions for protein synthesis and energy metabolism. Other host functions essential for Mu development have not yet been well characterized.

Mutants of $E$. coli ( $\mathrm{him}$ ) which prevent integration of phage lambda into the host chromosome were isolated by their ability to survive infection with a $\lambda \mathrm{N}^{-}$intc phage which kills cells in which it integrates (3). Strains containing himA mutations, which are located at $38 \mathrm{~min}$ on the $E$. coli map (4), are defective in $\lambda$ integration for two reasons. They produce reduced levels of $\lambda$ integrase protein (5), and they are defective in one subunit of the integration

\footnotetext{
${ }^{1}$ Present address: Cancer Research Unit, University of Western Ontario, London, Ontario N6A 5B7, Canada.

${ }^{2}$ Present address: Department of Molecular Biology, University of California, Berkeley, Calif. 94720.

${ }^{3}$ Author to whom reprint requests should be addressed.
}

host factor which participates directly in the $\lambda$ integration reaction (6). The $\operatorname{him} B$ mutation was originally isolated as a himB himC double mutant and is located at $82 \mathrm{~min}$ in or near gyrB. It does not block normal $\lambda$ integration or excision but is defective for $\lambda$ excision from a secondary att site (4).

The him $A$ and $\operatorname{him} B$ mutations also inhibit the growth of $\mathrm{Mu}$ (4). Mu development is severely inhibited in the himA host resulting in the production of very few phage and little host cell death ( 7 , R. K. Yoshida and M. M. Howe, manuscript in preparation). In contrast, in the $\operatorname{himB}$ host Mu growth is only partially inhibited, resulting in normal cell death and the generation of pinpoint plaques arising by delayed production of the phage burst (R. K. Yoshida and M. M. Howe, manuscript in preparation). To help determine the mechanism of growth inhibition in these hosts we mutagenized lysates of the heat inducible phage $\mathrm{Mu}$ cts62 (8) by growth ( 9 , slightly modified) in the mutD host KD1067 (10) and selected for $\mathrm{Mu}$ cts62 $n u A \mathrm{mu}-$ tants able to form plaques on lawns of a $\operatorname{himA42}$ host (4) and $\mathrm{Mu}$ cts62 nuB mutants able to form large plaques on lawns of a himB114 host (4) on TCMG (11) plates at $37^{\circ}$. Both $n u A$ and $n u B$ mutant phages arose at frequencies of $10^{-6}$ to $10^{-9}$ per plaque-forming phage.

The $n u$ mutations were located on the 
genetic map of $\mathbf{M u}$ by deletion mapping by marker rescue from $\lambda \mathrm{pMu}$ transducing phages (12) and from hosts with partially deleted $\mathrm{Mu}$ prophages (8). Since rescue of the $n u^{+}$allele was not directly selectable, amber mutations were introduced into the Mu nu phages by recombination or mutD mutagenesis $(9)$; then in the marker rescue assay $\mathrm{am}^{+}$recombinants were selected and scored for corescue of the $n u^{+}$allele.

The results shown in Fig. 1 demonstrate that nuA mutations are located within or to the left of gene $A$ at two distinguishable sites. The wild type alleles of the $n u A 1$ and nuA3 mutations can be rescued from prophage deletion strain MH165 (8) but not from strain KMBL1646 (13) whereas the wild type alleles of $n u A 106$ and $n u A 107$ cannot be rescued from either strain. The absence of $n u^{+}$rescue from strain KMBL 1646, which carries a deletion ending between $A 1504$ and $A 1093$ indicates that the $n u A$ alleles are located to the left of $A 1093$; however the absence of knowledge concerning the precise location of either endpoint of the deletion in strain MH165 makes it impossible to locate the mutations more definitively from this data.

The results of deletion mapping of the $n u B$ mutations by marker rescue from $\lambda \mathrm{pMu}$ transducing phages (12) and prophage deletion strains $(8,12)$ (Fig. 2) demonstrate that the $n u B$ mutations are located in the late region of $\mathrm{Mu}$ within or between $\mathrm{Mu}$ genes $G$ and $I$. The wild type
MU DNA IN DELETED LYSOGENS

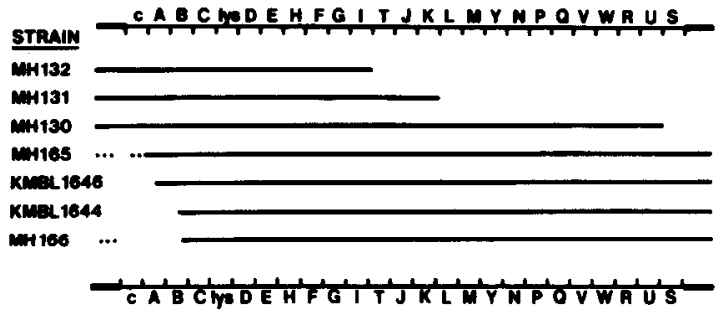

PERCENT CO-RESCUE OF $\mathrm{nu}^{+}$With am+ ALLELE

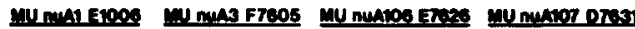

$$
\begin{gathered}
100(48) \\
\cdots . \\
\cdots \\
11(710) \\
<0.2(432) \\
<2.1(48) \\
<2.1(46)
\end{gathered}
$$

$$
\begin{gathered}
73(40) \\
87(48) \\
100(40) \\
<0.2(432) \\
<1.0(96) \\
-.- \\
. .
\end{gathered}
$$

$57(46)$
$52(46)$
$91(40)$
$<0.2(500)$
$<0.3(322)$
$\ldots$.
$<0.7(130)$

FIG. 1. Deletion mapping of $n u A$ mutations. The genetic map of the $\mathrm{Mu}$ prophage with genes $c$ through $U$ is given at the top left of the figure. The Mu DNA remaining in each prophage deletion strain is indicated by the horizontal line below the map. The dotted lines indicate that the exact endpoints of the deletions are unknown. The thick lines at the ends of the Mu DNA represent host DNA sequences immediately adjacent to the prophage. The reported locations of deletion endpoints in the MH (8) and KMBL (18) strains were based on marker rescue with only a few mutations in each gene. More detailed marker rescue analysis with amber mutants described by O'Day et al. (12) showed that the deletion endpoint in KMBL1646 was located between A1504 and A1093 and the endpoint in KMBL1644 was located between $B 5150$ and the pair $B 5175$ and $B 5176$. The rightmost deletion endpoint in strain MH165 was located to the left of the leftmost Aam mutations. (1791 and 7302); however, the locations of the leftmost deletion endpoints in strains MH165 and MH166 are unknown. They were originally selected as trimethoprim-resistant mutants to isolate deletions between thyA and a Mu inserted in lys (8); however, since they revert to thy $y^{+}$they do not contain such a deletion. Thus, their left deletion endpoints are unknown and could be within either Mu or host DNA. Two amber mutant derivatives of each of $4 \mathrm{Mu}$ cts62 phages carrying independently isolated nuA mutations were used to locate the nuA mutations by selecting for rescue of the $\mathrm{am}^{+}$ marker and scoring for corescue of the $n u^{+}$allele. Spot marker rescue assays (modified from 14) were performed, and the resulting areas of lysis were resuspended in SM diluting fluid (8) and plated for single plaques on lawns of the $\mathrm{Su}^{-}$host on TCMG (11) plates at $37^{\circ}$. Individual $\mathrm{am}^{+}$ plaques were resuspended in SM and spotted onto lawns of strains $\mathrm{K37}\left(\mathrm{him}^{+}\right.$) and $\mathrm{K634}$ (himA42) (4) on TCMG plates which were scored for phage growth after overnight incubation at $37^{\circ}$. The values given in the right portion of the figure indicate the percentage of $\mathrm{am}^{+}$recombinants which also rescued the $\mathrm{nu}^{+}$allele for one representative amber mutant for each phage. Numbers in parentheses indicate the total number of $\mathrm{am}^{+}$recombinants tested. Dashes indicate that the particular phage/host combination was not tested. 


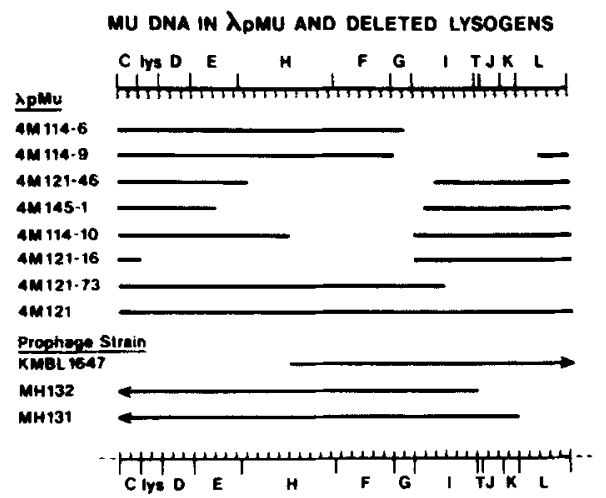

\begin{tabular}{|c|c|c|c|c|c|c|c|}
\hline & & CENT CO & RESCUE & nu+ WITh & $\mathrm{am}^{+}$ALL & & \\
\hline Mu r & & & & Mun $n$ & 3103 & Mu nut & 105 \\
\hline Eropo & (17013) & F7e?0 & J7621 & F7625 & 17824 & $1 \times 887633$ & $K 7635$ \\
\hline 0.8 (133) & -.. & $<1.0(06)$ & ... & $<1.0(96)$ & $-\cdot$ & $<0.1(799)$ & -8 \\
\hline$\cdots$ & ... & $<2.1(48)$ & ... & ... & $\cdots$ & $0.2(478)$ & $\cdots$ \\
\hline -.. & $<1.4(72)$ & $\cdots$ & $<2.1(48)$ &.- & -- & $<1.0(96)$ & $<0.6(178)$ \\
\hline ... & $<0.7(143)$ & -.- & $<1.1(04)$ & -.. & $<1.0(96)$ & $<0.1(960)$ & $<0.2(479)$ \\
\hline -.. & -- & --- & $\ldots$ & $\ldots$ & $\ldots$ & $<0.1$ (1051) & $\cdots$ \\
\hline ... & $5.6(72)$ & -.- & $7.3(90)$ & -.. & $6.2(96)$ & & $5.0(240)$ \\
\hline $13(16)$ & $\cdots$ & $13(24)$ & $\cdots$ & $40(98)$ & $\cdots$ & $14(96)$ & $\cdots$ \\
\hline $71(46)$ & $54(46)$ & $\cdots$ & $65(4 \theta)$ & - & $75(4 \theta)$ & $14(96)$ & $\cdots$ \\
\hline $94(40)$ & $38(40)$ & $73(48)$ & $21(48)$ & $94(47)$ & $67(4 B)$ & -.. & $31(48)$ \\
\hline $80(30)$ & $\cdots$ & $71(48)$ & $\cdots$ & $77(48)$ & $\cdots$ & $37(30)$ & $\therefore$ \\
\hline-- & --- & $\therefore$ & $48(48)$ & $\therefore$ & -.. & $\cdots$ & $60(48)$ \\
\hline
\end{tabular}

FIG. 2. Deletion mapping of $n u B$ mutations. The top left portion of the figure depicts the map of the central region of $\mathrm{Mu}$ (12) containing genes $C$ through $L$; each small subdivision represents one deletion interval and the large subdivisions indicate the genes. The Mu DNA carried by each $\lambda \mathrm{pMu}$ transducing phage $(12)$ or prophage deletion strain $(8,12)$ is indicated by the horizontal lines. The arrows for the prophage deletion strains indicate that the strain contains additional Mu DNA in the direction to which the arrow points. Two amber mutant derivatives, one on each side of the $G-I$ region, were isolated for each of four independently isolated Mu cts62 nuB mutant phages and were used as described in the legend to Fig. 1 for $\mathrm{am}^{+}$spot marker rescue analysis followed by scoring for corescue of the $n u^{+}$allele by growth on strain $\mathrm{K} 760$ ( $\mathrm{Him}^{+} \mathrm{Su}^{+} \operatorname{dctA}$ ilv his pps gal strA) but not on strain $\mathrm{K} 761$ ( $h i m B 114 \mathrm{Su}^{+} \operatorname{dct} A$ his $p p s$ gal strA). The method of presentation of results is described in the legend to Fig. 1.

alleles of all four $n u B$ mutations were rescued from $\lambda$ pMu 4M121-16 but not from $\lambda \mathrm{pMu} 4 \mathrm{M} 145-1$. $\lambda \mathrm{pMu} 4 \mathrm{M} 121-16$ is deleted for wild type alleles of all known $G$ amber mutations but allows rescue of all $I$ amber mutations; while $\lambda \mathrm{pMu} 4 \mathrm{M} 145-1$ is deleted for the DNA corresponding to all $G$ amber mutations and the adjacent $6 I$ mutations (12).

Marker rescue of the $n u B 105$ phage with $\lambda \mathrm{pMu} 114-9$ gave the unexpected result of $0.2 \%$ rescue $\left(n u^{+} / \mathrm{am}^{+}\right)$despite the absence of the $G-I$ region in the latter phage. This was based on finding $1 n u^{+}$plaque among $478 \mathrm{am}^{+}$recombinants but was not confirmed in tests of 1143 additional $\mathrm{am}^{+}$ recombinants which were all $n u B$. Examination of the results with other phages, particularly with $\lambda \mathrm{pMu}$ 114-6 which carries even more $\mathrm{Mu}$ DNA than $\lambda$ pMu 1149 but still does not allow rescue of $n u^{+}$, led us to disregard the single plaque observed with $\lambda$ pMu 114-9.

The location of $n u$ mutations is consistent with the properties of $\mathrm{Mu}$ in the him hosts. Mu development in the himA host is blocked early before cell killing can occur (7) and the $n u A$ mutations are located in the region of $\mathrm{Mu}$ which is transcribed early (15). Similarly, $\mathbf{M u}$ growth in the $\operatorname{him} B$ host causes normal levels of cell death and a delayed phage production (R. K. Yoshida and M. M. Howe, manuscript in preparation), presumably by inhibiting a later step in phage development, and the $n u B$ mutations are located in a region transcribed later. The precise nature of the block to $\mathrm{Mu}$ development in these hosts is not yet clear. Mu integration in the himA host is normal $(\gamma ; \mathrm{R}$. K. Yoshida and M. M. Howe, manuscript in preparation) but DNA synthesis (B. Waggoner and $M$. Pato, personal communication) and late RNA synthesis (R. K. Yoshida and M. M. Howe, manuscript in preparation) are blocked. The $\operatorname{him} A$ gene product might participate directly in Mu DNA replication and only indirectly affect late transcription, or conversely, it might be necessary for transcription of genes and/or sites which are required for replication. Alternatively, transposition of Mu subsequent to the initial integration event might require him $A$ gene product and thus result in the requirement for $\operatorname{him} A$ for DNA replication. If the $h i m B$ mutations are altered in one of the subunits of DNA gyrase, as is suggested by their linkage to $\operatorname{gyr} B(4)$, 
they might exert their effect on $\mathrm{Mu}$ via altered transcription, delayed replication, or delayed maturation of the phage particles. Further experiments to define the roles of himA and himB in Mu development and the alterations present in the $n u A$ and $n u B$ mutants are in progress.

\section{ACKNOWLEDGMENTS}

This work was supported by the College of Agricultural and Life Sciences, University of Wisconsin, Madison, Wisconsin, and by grants AI-12731 to M.M.H. and AI-14363 to D.I.F. from the National Institue of Allergy and Infectious Diseases and grant NP-264 to M.M.H. from the American Cancer Society. R.K.Y. was supported by training grant GM-07215 from the National Institute of General Medical Sciences. M.M.H. is the recipient of Research Career Development Award AI-00274 from the National Institute of Allergy and Infectious Diseases.

\section{REFERENCES}

1. Toussaint, A., and Fablen, M., Molec. Gen. Genet. 131, 209-214 (1974).

2. Toussaint, A., and LeCoCQ, J., Molec. Gen. Genet. 129, 185-188 (1974).
3. Miller, H. I., and FriedmaN, D. I., In "DNA Insertion Elements, Plasmids and Episomes" (A. I. Bukhari, J. Shapiro, and S. Adhya, eds.), pp. 349-356. Cold Spring Harbor Laboratory, New York, 1978.

4. MLLeR, H. I., KIKUChI, A., NASH, H. A., WeISBERG, R. A., and FRIEDMAN, D. I., Cold Spring Harbor Symp. Quant. Biol. 43, 1121-1126 (1979).

5. Miller, H. I., Cell 25, 269-276 (1981).

6. MILLER, H. I., and NASH, H. A., Nature (London) 290, 523-526 (1981).

7. Miller, H. I., and Friedman, D. I., Cell 20, 711719 (1980).

8. Howe, M. M., Virology 54, 93-101 (1973).

9. HowE, M. M., O'DAY, K. J., and SCHULTZ, D. W., Virology 93, 303-319 (1979).

10. Fowler, R., DeGnen, G., and Cox, E., Molec. Gen. Genet. 133, 179-191 (1974).

11. SChumM, J. W., MOORE, D. D., BlatTwer, F. R., and Howe, M. M., Virology 105, 185-195 (1980).

12. O'DAY, K. J., Schultz, D. W., ERICSEN, W., RAWLUK, L. B., and Howe, M. M., Virology 93, 320328 (1979).

13. Wijffelman, C. A., Westmaas, G. C., and van DE PUTTE, P., Virology 54, 125-134 (1973).

14. HowE, M. M., SCHUMM, J. W., and TAYLOR, A. L., Virology 92, 108-124 (1979).

15. WiJfFelman, C., and VAN DE PUTTE, P., Molec. Gen. Genet. 135, 327-337 (1974). 